\title{
Form deviations caused by lateral displacement errors in annular subaperture stitching interferometry
}

\author{
Markus Schake $\odot,{ }^{\mathrm{a}, *}$ Jörg Riebeling $\odot$,, and Gerd Ehret $\oplus^{\mathrm{a}}$ \\ ${ }^{a}$ Physikalisch-Technische Bundesanstalt, Braunschweig, Germany \\ ${ }^{b}$ Universität Kassel, FB 16 Elektrotechnik/Informatik Messtechnik, Kassel, Germany
}

\begin{abstract}
Annular subaperture stitching interferometry (ASSI) is a common approach for the measurement of aspherical surfaces. A common obstacle of ASSI is the occurrence of lateral displacement errors when the sensor or specimen is repositioned between the subaperture measurements. Our contribution focuses on modeling of the statistical displacement errors. A virtual experiment is presented simulating the propagation of the displacement errors through a cumulative and a global stitching algorithm to the retrieved surface form. For the considered experimental setup, the uncertainty in lateral position depends on the positioning uncertainties of the employed motion system and the uncertainty in the absolute distance measurement between the sensor and specimen. The lateral displacement uncertainty is determined experimentally employing a calibratable lateral grating. Thus, it is traceable to the SI unit of the length (meter). The experimental results show that the lateral displacement errors may be modeled by a normal distribution, and the results of the virtual experiment indicate that the statistical lateral displacement errors transfer linear through the stitching procedure and also cause a normal distributed topography error. This enables the assignment of an expanded uncertainty to each individual sample point employing the Zernike polynomial expression of the topography measurement. (C) The Authors. Published by SPIE under a Creative Commons Attribution 4.0 Unported License. Distribution or reproduction of this work in whole or in part requires full attribution of the original publication, including its DOI. [DOI: 10.1117/1.OE.59.12.124105]
\end{abstract}

Keywords: annular subaperture; stitching; interferometry; traceability; measurement uncertainty.

Paper 20201156 received Oct. 1, 2020; accepted for publication Nov. 30, 2020; published online Dec. 14, 2020.

\section{Introduction}

To receive traceable and comparable measurement results, it is mandatory to provide a statement of uncertainty achieved with the instruments and algorithms employed in the measurement process. The design and fabrication of traceable calibration artifacts for optical 3D surface topography measurements is challenging, due to the great variety of applications and specimen geometries. National metrology institutes are working on primary instruments and calibration artifacts to provide the basis of traceable uncertainty statements in industrial applications. ${ }^{1,2}$ A universal calibration artifact comprising six different material measures with varying scales for the holistic calibration of an optical instrument employing a single specimen is proposed in Ref. 1. The artifact covers typical metrological properties listed in the ISO 25178-600 standard and shows good producibility and aging behavior. The national metrology institute of Germany, Physikalisch-Technische Bundesanstalt (PTB), recently proposed a multispherical artifact for traceable calibration of form measurements of freeform surfaces, ${ }^{3}$ which may foster the standardization of uncertainty statements for the measurand of the associated form measuring instruments. In addition to the availability of traceable calibration artifacts to characterize the measurement uncertainties related to the instruments, a meaningful statement of uncertainty also requires profound knowledge about uncertainties introduced by the calculations and algorithms involved in the translation of the recorded images to the surface topography. The uncertainty analysis of the algorithm may be based on an analytic modeling of the signal processing as, e.g., in the contribution of Henning and Guisca from the National Physical Laboratory,

*Address all correspondence to Markus Schake, markus.schake@ptb.de 
which describes the uncertainties associated with topography retrieval by means of Fourier domain analysis. ${ }^{4}$ Another emerging method for sensitivity analysis is Monte-Carlo method, which models the uncertainty of input quantities as a random distribution and yields a confidence interval for the uncertainty of the dependent measurand by means of a virtual experiment. $^{2}$ The Monte-Carlo approach is demonstrated by virtual experiments regarding topography measurements with a tilted wave interferometer. ${ }^{5}$ In a research cooperation of the PTB and the University of Kassel considering form measurements of rotationally symmetric, spherical, and aspherical surfaces, the capability of a dynamically aligned interferometric line sensor to work as a traceable instrument for calibration is tested. The topography measurement system comprising an interferometric line sensor, two fiber coupled interferometric point sensors, and a complex motion system is continuously enhanced and developed. Advances, measurements, and characteristics of the setup have been reported, which put detailed emphasis on the interferometric sensors and the rotation stages run-out compensation ${ }^{6-8}$ as well as the employed stitching algorithms. ${ }^{9,10}$ This contribution focuses on the traceable characterization of the lateral displacement errors occurring in the system during the repositioning of the sensor between subaperture measurements. The uncertainty in lateral position is determined employing a lateral grating as a calibration artifact. A model of the lateral displacement uncertainty is introduced and compared to statistical deviations of the subaperture measurements. The propagation of the lateral displacement uncertainty through the surface stitching algorithms is analyzed employing a Monte-Carlo study. The resulting uncertainty in the surface topography is determined by virtual experiments.

\section{Experimental Setup and Algorithms}

This section starts with a short introduction of the interferometric measurement setup and the calibration routine to determine the lateral displacement uncertainty. Then the employed cumulative and global stitching algorithms are shortly outlined, describing their dependence on the lateral displacement uncertainty.

\subsection{Interferometer and Positioning System}

The interferometric line sensor is set up as a Michelson interferometer with low coherent green light-emitting diode (LED) illumination coupled from a high-intensity LED into the setup via a liquid light guide, which is visible on the left side in Fig. 1(a). The illumination source is separated from the interferometer to reduce the size of the actual sensor and to prevent thermal influences caused by the LED's power dissipation. The interferometric unit comprised of the microscope objective, the beam splitter, and reference arm is mounted in a single aluminum housing for mechanic stability. The optical axis of the reference and measurement path are indicated emerging from the beam splitter in Fig. 1(a) and labeled $L_{\text {ref }}$ and $L_{\mathrm{m}}$, respectively. The center of rotation of the rotary stage B is marked by the red solid circle and its distance to the beam splitter cube is labeled $L_{\text {rot }}$. The dashed red lines indicate the beam paths of the interferometer when tilted by the angle $\alpha_{k}$ in the $x-z$ plane. The instrument is dedicated to the measurement of rotationally symmetric objects. Therefore, it is convenient to address the lateral coordinates by the radial distance $r_{k}$ [see Eqs. (4) and (5)] to the center of rotation of the lower rotary stage $\mathrm{C}$, which rotates the specimen around the optical axis by the azimuthal angle $\theta$ in the $x-y$ plane. Before the measurement of each annular subaperture, the measurement position is iterated by the distance $x_{\mathrm{it}, k}$ and the interferometer is tilted according to the local slope of the specimen at the current radial position, such that the optical axis is oriented normal to the surface. Rotation of the interferometer causes a change $\Delta x_{k}$ in the lateral coordinate and a change $\Delta L_{k}$ in the absolute axial distance between the specimen and the center of rotation. The change $\Delta L_{k}$ is compensated by a depth scan of the interferometer employing the envelope of the low coherent interferogram to align the sensor in the path length equilibrium condition $L_{\mathrm{m}}=L_{\mathrm{ref}}$ with respect to the specimen surface. This ensures a constant distance between the specimen and the beam splitter at the start of each subaperture measurement with the axial measurement uncertainty of the Michelson interferometer. The lateral displacement $\Delta x_{k}$ is compensated by motion of the $x$-axis. However, the motion distance depends on the absolute distance $L_{\text {ref }}+L_{\text {rot }}$, 


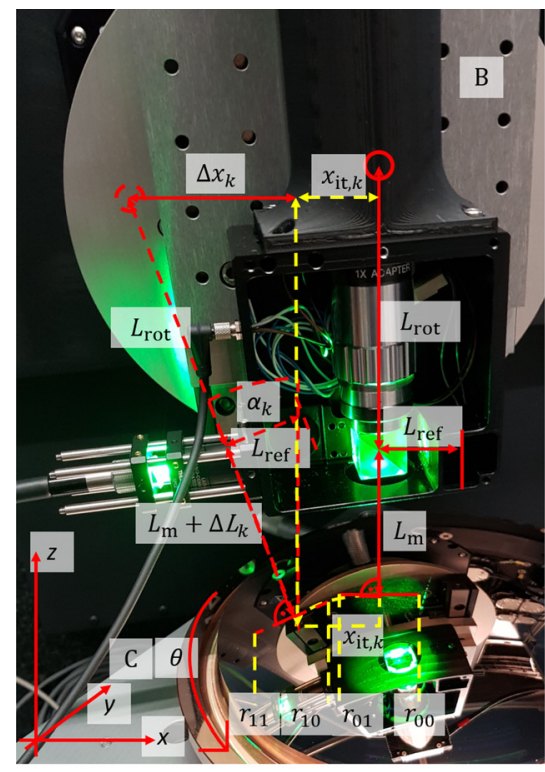

(a)

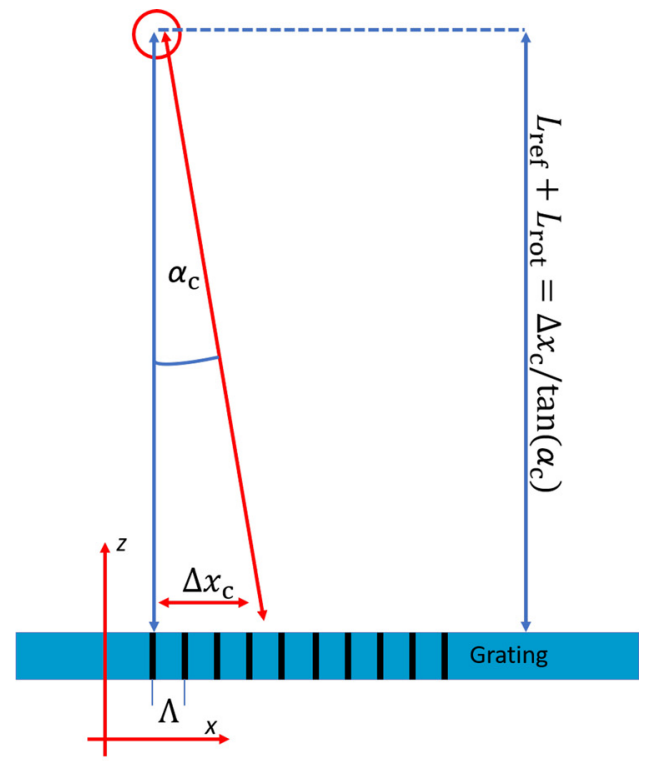

(b)

Fig. 1 (a) Photography of the employed Michelson interferometer. With $x, y$, and $z$ orientation of the global coordinate system, $L_{m}$ distance to the specimen, $L_{\text {ref }}$ distance to the reference plane, $L_{\text {rot }}$ distance to the center of rotation of rotary stage $B, \Delta L_{k}$ distance change to the specimen when rotating, $\Delta x_{k}$ lateral displacement when rotating, $\alpha_{k}$ angle of rotation, $x_{i t, k}$ lateral displacement between subaperture measurements, $r_{k 0}, r_{k 1}$ radial boundaries of the $k$ 'th subaperture, $\theta$ azimuthal angle of rotary stage $C$. (b) Schematic drawing of the lateral displacement $\Delta x_{\mathrm{c}}$ when rotating the sensor by the calibration angle $\alpha_{\mathrm{c}}$ employing a calibratable grating of lateral period $\Lambda$ as a calibration artifact.

which has to be calibrated. The calibration procedure is described in Ref. 9, and it is illustrated in Fig. 1(b) and summarized here shortly with an extension in terms of signal analysis to reduce the measurement uncertainty.

- A calibratable lateral grating is employed as the specimen.

- The interferometer is aligned perpendicular to the grating $\alpha_{\mathrm{c}}=0^{\circ}$ and positioned in the path length equilibrium condition $L_{\mathrm{m}}=L_{\mathrm{ref}}$ via a low coherent interferometry scan.

- The reference arm is blocked. The detector records a microscopic image of the grating without interference fringes.

- The interferometer is tilted by the angle $\alpha_{\mathrm{c}}$ and a temporal series of images is recorded where each image shows an increased phase shift in the periodical grating.

- The phase shift between the consecutively recorded images of the grating is retrieved employing a Fourier transform approach. In the spectral domain, the phase information is shifted to the frequency peak associated with the grating period, similar to a carrier fringe approach in interferometry. Employing a lock-in detection approach, ${ }^{8,11}$ one phase value is retrieved from each recorded line image.

- The sum over all recorded phase shifts yields the lateral displacement $\Delta x_{\mathrm{c}}$ with an expected uncertainty $u_{x, \mathrm{c}}$ of approximately $\Lambda / 360$.

- Employing the formula for the propagation of uncertainty in Eq. (1) yields an estimate of the expected uncertainty $u_{L}$ in the calibrated absolute distance $L_{\text {ref }}+L_{\text {rot }}$.

- A series of $N=1000$ calibration measurements employing a calibratable grating with a period $\Lambda=100 \mu \mathrm{m}$ and $\alpha_{\mathrm{c}}=1^{\circ}$ yields a mean lateral displacement of $\overline{\Delta x_{\mathrm{c}}} \approx 3.007 \mathrm{~mm}$ with a standard uncertainty ${ }^{12}$ of $u_{x, \mathrm{c}} \approx 0.34 \mu \mathrm{m}$. The lateral displacement measurement results are depicted in Fig. 2(a) and the associated histogram is depicted in Fig. 2(b) together with a normal distribution employing $u_{x, \mathrm{c}} \approx 0.34 \mu \mathrm{m}$ (solid orange line). Figure 2(c) shows the cumulative empirical distribution (blue circles) in comparison to the cumulative normal distribution function (orange line). The good alignment of the 


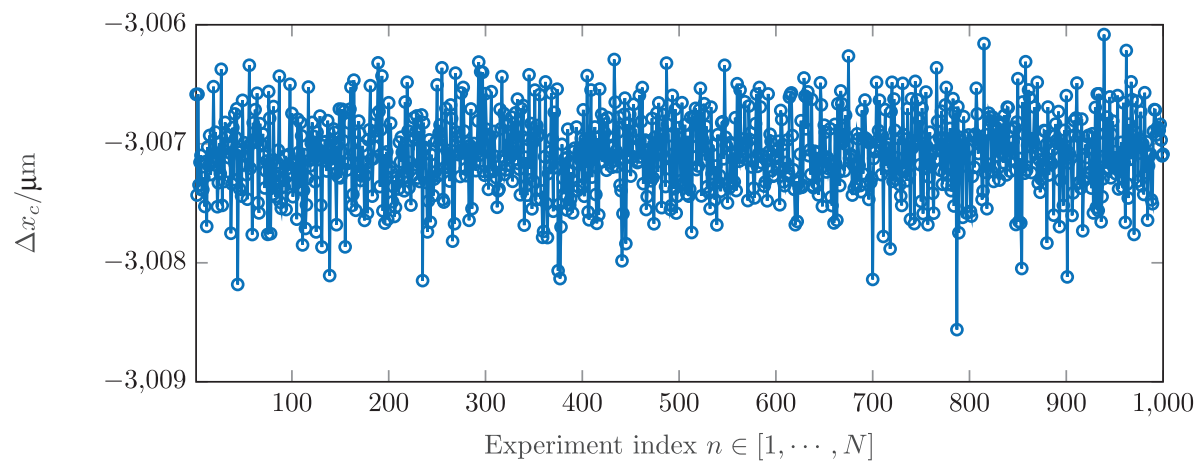

(a)

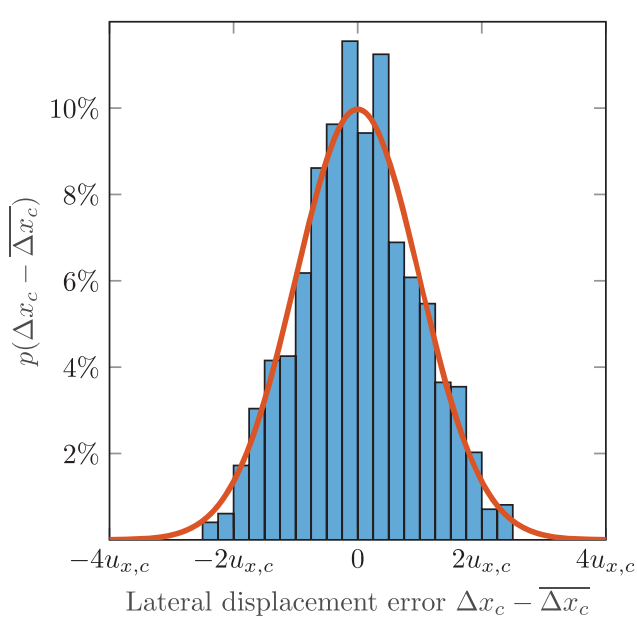

(b)

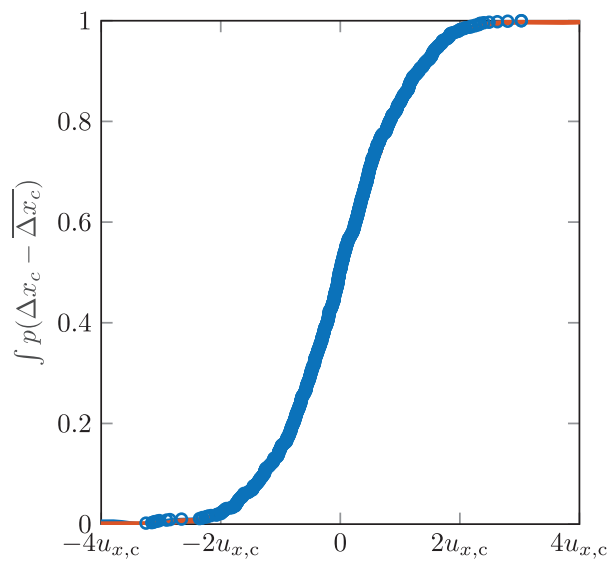

Lateral displacement error $\Delta x_{c}-\overline{\Delta x_{c}}$

(c)

Fig. 2 (a) Lateral displacement $\Delta x_{\mathrm{c}}$ measured in $N=1000$ calibration experiments in the path length equilibrium distance $L_{\mathrm{m}}=L_{\text {ref }}$ (b) Histogram of the $N=1000$ samples compared to the probability density function of a normal distribution (solid orange line) with standard deviation $u_{x, \mathrm{c}} \approx 0.34 \mu \mathrm{m}$. (c) Cumulative empirical distribution function (blue circles) and cumulative distribution function of the simulated normal distribution (solid orange line).

cumulative distribution functions indicates that the statistical lateral displacement errors occurring when calibrating the system may be feasible modeled as a normal distribution. The rotation axis $B$ is specified by an angular standard uncertainty of $u_{\alpha} \approx 1^{\prime \prime}$ by the acceptance test procedure for axis accuracy. From this experiment, the absolute distance between the specimen and the center of rotation of the $B$-axis in the path length equilibrium condition is determined as $L_{\text {ref }}+L_{\text {rot }} \approx 172.3 \mathrm{~mm}$ with a combined standard uncertainty ${ }^{12}$ of $u_{L} \approx 20 \mu \mathrm{m}$.

$$
u_{L}=\sqrt{\left(u_{x, \mathrm{c}} \cdot \frac{1}{\tan \alpha_{\mathrm{c}}}\right)^{2}+\left(-\Delta x_{\mathrm{c}} \cdot u_{\alpha} \frac{1}{\sin \alpha_{\mathrm{c}}^{2}}\right)^{2}} .
$$

The actual interferometric annular subaperture measurement starts when the sensor is aligned perpendicular to the specimen in the path length equilibrium distance facing the radial interval $r_{k}=\left[r_{k 0}, r_{k 1}\right]$ with $k \in 1, \cdots, K$ indicating the index of the subaperture. The rotational stage $\mathrm{C}$ rotates the specimen beyond the line sensor and the topography of the annular subaperture is retrieved employing a temporal carrier fringe approach. For this purpose, the interferometers reference mirror is mounted on an oscillation coil, which is actuated by a driving voltage with a frequency of $1 \mathrm{kHz}$. The employed line camera records images at a rate of up to $100 \mathrm{kHz}$, sufficiently oversampling the path length modulated interference images. The path length modulation shifts the topography-dependent phase information of the interferogram to the modulation 
Table 1 Estimate of spatial wavelength regimes associated with the surface texture elements of from, waviness and roughness dependent on the specimen diameter $D$. Fitting the topography into Zernike polynomials has a strong low-pass filtering effect, the transfer function of the Zernike polynomials with $L=36$ coefficients shows a suppression of $-40 \mathrm{~dB}$ for topography features with a frequency of $\approx 25 \mathrm{~Hz}$.

\begin{tabular}{lc}
\hline \hline Surface texture element & Spatial wavelength regime $\Lambda_{s}$ \\
\hline Form & $D \cdot 10^{0}>\Lambda_{s}>D \cdot 10^{-2}$ \\
Waviness & $D \cdot 10^{-2}>\Lambda_{s}>D \cdot 10^{-4}$ \\
Roughness & $D \cdot 10^{-4}>\Lambda_{s}>D \cdot 10^{-6}$ \\
\hline \hline
\end{tabular}

frequency peak in the spectral domain. This enables the application of the noise robust lock-in detection approach ${ }^{8,11}$ to each pixel of the line sensor to retrieve the topography of the scanned annular subaperture. The topography retrieval based on temporal carrier fringes limits the data rate of the sensor to two height values per oscillation period of the path length modulating reference arm. Thus, with the current specifications, the maximum data output of the line sensor is 2000 points per second for each pixel. However, the rotationally symmetric specimens are expected to show no significant form variations in the azimuthal direction. For the form measuring purpose, it is sufficient to achieve a resolution in the range of $[0.1,1 \mathrm{~mm}]$ in the azimuthal direction. Structures of higher lateral frequency are potentially averaged during the scanning procedure or canceled out during the stitching procedure, which employs Zernike polynomial fitting. It is noteworthy that there is no general absolute criterion to separate between form, waviness, and roughness. The wavelength regime associated with these surface texture elements depends on the specimen and its intended application. ${ }^{13}$ To yield an estimate of the considered wavelength regime, the specimen's diameter $D$ may be considered and the surface texture elements of form, waviness, and roughness may each be associated with two decades of decreasing feature size starting from the dimension of the diameter. The experimental setup at PTB is suitable to mount specimens with a diameter of multiple hundred millimeters. Considering this and aiming at a sufficient oversampling yield the lateral resolution limits required for the form measuring setup outlined above. The camera line contains 4096 pixels with a pixel size of $10.56 \mu \mathrm{m} \times 10.56 \mu \mathrm{m}$, thus employing a $5 \times$ microscope objective covering a length of $\left|r_{k 1}-r_{k 0}\right|_{\perp} \approx 8.65 \mathrm{~mm}$ oriented perpendicular to the specimen surface. A specimen with a diameter of $D=300 \mathrm{~mm}$ contains at least $K \geq \operatorname{round}\left(D /\left(2\left|r_{k 1}-r_{k 0}\right|_{\perp}\right)\right)=17$ subapertures. The outer perimeter of the specimen is $D \cdot \pi \approx 942.5 \mathrm{~mm}$. Employing a lateral sampling interval of $0.1 \mathrm{~mm}$ with a sample rate of $2 \mathrm{kHz}$ results in a rotation frequency of the specimen of $\approx 0.2 \mathrm{~Hz}$ or a rotation velocity of $\approx 70 \mathrm{deg} / \mathrm{s}$. Considering a constant rotation speed for all subapertures, this results in an overall measurement time of $\approx 85 \mathrm{~s}$. This is a simplified example, which does not consider the decrease in surface coverage by the line sensor with increasing slope of the specimen and also ignores the time required for repositioning of the sensor between subapertures and the overlap between consecutive subapertures. However, it indicates that the expected time for topography measurement will be in the range of several minutes (Table 1).

\subsection{Cumulative and Global Stitching}

Due to the repositioning of the sensor between the consecutive recording of annular subapertures, each subaperture is recorded in a local coordinate system. To retrieve the full topography, the annular subapertures are stitched together referring to a global set of coordinates. The experimental setup introduced in Sec. 2.1 employs a planar reference wavefront. Therefore, the possible occurring wavefront aberrations due to positioning errors are sufficiently described by the three Zernike coefficients of piston and two tilts. ${ }^{9,10,14-17}$ The stitching algorithm is based on the solution of an optimization problem similar to those in Refs. 14, 16, and 17 and employs a machine learning-based Zernike polynomial fitting ${ }^{18}$ to fit the annular subapertures before proceeding with the cumulative or global stitching method. 
The cumulative stitching requires overlapping regions between the consecutively recorded subapertures. The low-order translational wavefront aberrations between the subapertures are fitted into a three coefficient annular Zernike polynomial, which than is subtracted from the subaperture to be stitched. This process is repeated starting at a chosen reference aperture until all subapertures are stitched together. The correction polynomials are accumulated over the subapertures, such that the last subaperture to be stitched undergoes all corrections of piston and tilt, which its predecessors have been subject to. However, assuming a plane reference wavefront, a nonlinear accumulation of translational errors is precluded since the piston and tilt coefficients of the correction planes are constant on the unit circle. If there is an error in the correction plane employed to stitch the first and second subapertures, it will affect all following subapertures in the same way and has no influence on their relative orientation. When the overlapping areas of all subapertures are aligned by removing the piston and tilt aberrations, the resulting surface is fitted into a single Zernike polynomial defined in the global coordinate space. A simulation example of the cumulative stitching and further details concerning the use of a plane reference wavefront to omit higher order wavefront aberrations induced by alignment errors are reported in Ref. 9.

The global stitching algorithm is reported in Ref. 10, putting emphasis on the fast and convenient formulation of the optimization problem. Other than the cumulative stitching, the global stitching algorithm does not require any overlapping areas between the recorded subapertures. It also exploits the fact that the wavefront aberrations related to the translational errors between subapertures for a plane reference wavefront are covered by the three low-order Zernike coefficients of piston and tilt. The stitched surface topography is described by the sum of a high-order Zernike polynomial with $(L-M)$ coefficients, which is defined in the global coordinate system and the translational errors described by $M$ Zernike coefficients each in their local coordinates.

$$
\underbrace{W(r, \theta)}_{\mathbf{Y}}=\underbrace{\sum_{k=1}^{K}\left[\sum_{i=1}^{M} b_{k i} Z_{k i}\left(r_{k}, \theta, k\right)+\sum_{i=M+1}^{L} B_{i} Z_{k i}\left(r_{k}, \theta, k\right)\right]}_{\mathbf{X} \cdot \mathbf{P}} .
$$

In Eq. (2), the global wavefront $W(r, \theta)=[\mathbf{Y}]_{K N_{\text {sub }} \times 1}$ represents the sampled surface topography with $N_{\text {sub }}$ sample points on each of the $K$ subapertures. The matrix representation of the Zernike polynomials evaluated at the supporting points is represented by $[\mathbf{X}]_{K N_{\text {sub }} \times(K M+(L-M))}$ and $[\mathbf{P}]_{(K M+(L-M)) \times 1}$ refers to the vector of the unknown local $b_{k i}$ and global $B_{i}$ Zernike coefficients. The Zernike polynomial coefficients describing the stitched surface in global coordinates result from solving the optimization problem as given in Eq. (3).

$$
\mathbf{P}=\left(\mathbf{X}^{T} \mathbf{X}\right)^{-1} \cdot \mathbf{X}^{T} \mathbf{Y}
$$

Details about the formulation of the global optimization problem in Eq. (2) and the matrices $\mathbf{Y}$, $\mathbf{X}$, and $\mathbf{P}$ are reported in Ref. 10 .

\section{Radial Displacement Uncertainty}

In the scope of this contribution, the measurement of rotationally symmetric specimens of unit radius $-1 \leq r \leq 1$ and azimuthal angle $0 \leq \theta \leq 2 \pi$ is considered. This is convenient because the Zernike polynomials are defined on the unit circle and the results obtained are easily scalable to any arbitrary specimen diameter $D$. A common feature of both the cumulative and the global stitching algorithm is their dependence on the radial and azimuthal position. To identify the overlapping regions for the cumulative stitching and correctly assert the supporting points in the matrix $\mathbf{X}$ in the optimization problem for the global stitching, each recorded sample requires an unambiguous allocation of $(r, \theta)$. As depicted in Fig. 1, the interferometric line sensor is repositioned between each subaperture measurement, such that its optical axis is aligned perpendicular to the best-fit line of the current surface interval in the path length equilibrium distance $L_{\mathrm{m}}=L_{\mathrm{ref}}$. Therefore, on the $k^{\prime}$ th subaperture, the radial position is given by $r \in r_{k}=\left[r_{k 0}, r_{k 1}\right]$. The boundaries of the $k$ 'th radial interval $r_{k 0}, r_{k 1}$ are calculated employing the lateral iterations $x_{\mathrm{it}, k}+u_{x, \mathrm{it}}$, the lateral displacement due to tilting of the sensor $\Delta x_{k}$, its associated standard uncertainty $u_{x, k} \approx \frac{\alpha_{k}}{\alpha_{\mathrm{c}}} u_{x, \mathrm{c}}$, the linear stage's starting position $x_{0}$, the 
nominal width of the field of view $\left|r_{k 1}-r_{k 0}\right|_{\perp} \approx 8.65 \mathrm{~mm}$, and the current tilt angle $\alpha_{k}+u_{\alpha}$ of the rotation stage $\mathrm{B}$.

$$
\begin{aligned}
& r_{k 0}=\left[\sum_{j=1}^{k}\left(x_{\mathrm{it}, j}+u_{x, \mathrm{it}}\right)+\Delta x_{k}+\frac{\alpha_{k}}{\alpha_{\mathrm{c}}} u_{x, \mathrm{c}}+x_{0}+u_{x, \mathrm{it}}\right]-\frac{1}{2}\left[\left|r_{k 1}-r_{k 0}\right|_{\perp} \cos \left(\alpha_{k}+u_{\alpha}\right)\right], \\
& r_{k 1}=\left[\sum_{j=1}^{k}\left(x_{\mathrm{it}, j}+u_{x, \mathrm{it}}\right)+\Delta x_{k}+\frac{\alpha_{k}}{\alpha_{\mathrm{c}}} u_{x, \mathrm{c}}+x_{0}+u_{x, \mathrm{it}}\right]+\frac{1}{2}\left[\left|r_{k 1}-r_{k 0}\right|_{\perp} \cos \left(\alpha_{k}+u_{\alpha}\right)\right] .
\end{aligned}
$$

The uncertainty $u_{x, \mathrm{it}}$ of the $x$-axis motion is specified by the distributor as $u_{x, \mathrm{it}} \approx 1 \mu \mathrm{m}$ and the uncertainty of the $B$-axis motion is specified as $u_{\alpha} \approx 1^{\prime \prime}$. The uncertainty $u_{x, \mathrm{c}}$ of the lateral displacement during calibration is scaled with $\frac{\alpha_{k}}{\alpha_{\mathrm{c}}}$ since the calculation of $\Delta x_{k}$ analog to $\Delta x_{\mathrm{c}}$, as depicted in Fig. 1(b), is based on the calibrated absolute distance $L_{\text {ref }}+L_{\text {rot }}+u_{L}$ and does not employ the precise Fourier domain evaluation used in the calibration procedure. The absolute distance between the center of rotation and the specimen and the associated combined uncertainty $u_{L}$ are the same during the experiment as in the calibration routine. However, the applied tilting angles $\alpha_{k}$ may exceed $\alpha_{\mathrm{c}}=1^{\circ}$ and $\Delta x_{k}$ may exceed $\Delta x_{\mathrm{c}}$. The linear scaling of the uncertainty yields a good estimate for angles $-10^{\circ} \leq \alpha_{k} \leq 10^{\circ}$. For greater tilt angles, the scaling in Eq. (6) should be applied, which employs the less restrictive approximation $\Delta x_{\mathrm{c}} \gg u_{x, \mathrm{c}}$ and $\Delta x_{k} \gg u_{x, k}$.

$$
\begin{aligned}
& L_{\mathrm{ref}}+L_{\mathrm{rot}}+u_{L}=\left(\Delta x_{\mathrm{c}}+u_{x, \mathrm{c}}\right) / \tan \left(\alpha_{\mathrm{c}}\right) \\
& L_{\mathrm{ref}}+L_{\mathrm{rot}}+u_{L}=\left(\Delta x_{k}+u_{x, k}\right) / \tan \left(\alpha_{k}\right) \\
& u_{x, k} \approx \frac{\tan \left(\alpha_{k}\right)}{\tan \left(\alpha_{\mathrm{c}}\right)} u_{x, \mathrm{c}} .
\end{aligned}
$$

Employing the formula for the propagation of uncertainty, the expected combined uncertainty for the borders of the radial intervals $u_{r, k}$ is given by Eq. (7).

$$
u_{r, k}=\sqrt{\left[(k+1) \cdot u_{x, \mathrm{it}}\right]^{2}+\left[\frac{\tan \left(\alpha_{k}\right)}{\tan \left(\alpha_{\mathrm{c}}\right)} \cdot u_{x, \mathrm{c}}\right]^{2}+\left\{\frac{1}{2}\left[\left|r_{k 1}-r_{k 0}\right|_{\perp} \sin \left(\alpha_{k}\right)\right] \cdot u_{\alpha}\right\}^{2}} .
$$

Since the distribution of the lateral displacement errors $\Delta x_{c}$ as depicted in Fig. 2(b) is sufficiently approximated by a normal distribution of standard deviation $u_{x, \mathrm{c}}$ and a normal distribution is also assumed for the axis errors, the radial interval errors are also expected to be distributed normally.

However, the model in Eq. (7) does only consider statistical errors of the setup. A violation of the sensor's positioning conditions, being aligned in the distance $L_{\mathrm{m}}=L_{\text {ref }}$ and perpendicular to the best-fit line of the surface section, also introduces a systematic error. This systematic error is partially visible in the interferograms measured after realignment on the subapertures and will influence the surface stitching when the recorded measurement data are assigned to the erroneous tracked radial position. The weighting of the $x$-axis uncertainty $(k+1) \cdot u_{x, \text { it }}$ is a worst case estimation, considering the unlikely case that the positioning errors introduced by the axis with each step have the same sign and add up.

Figure 3(a) illustrates the combined uncertainty $u_{r, k}$ for varying combinations of tilt angles over the number of subapertures. The statistical errors described by Eq. (7) are visible in the interferometric data recorded in each subaperture measurement. Considering an ideally spherical measurement object, the curvature is constant on the whole sphere and the interferogram recorded, when being aligned perpendicular to the best-fit line of a subaperture should look identically. However, the systematic and statistical misalignment errors may cause a tilt deviation between the interferograms. Employing the calibrated absolute distance $L_{\text {ref }}+L_{\text {rot }}$ and the width of the camera line $\left|r_{k 1}-r_{k 0}\right|_{\perp}$, the statistical lateral displacement error may be estimated directly from the unwrapped subaperture topography of the interferometric measurement. The measured tilt error is denoted as $e_{\alpha, k}$ and based on the height difference between the edges of each subaperture $z_{k}$ after subtracting the mean subaperture height $\overline{z_{k}}$. 


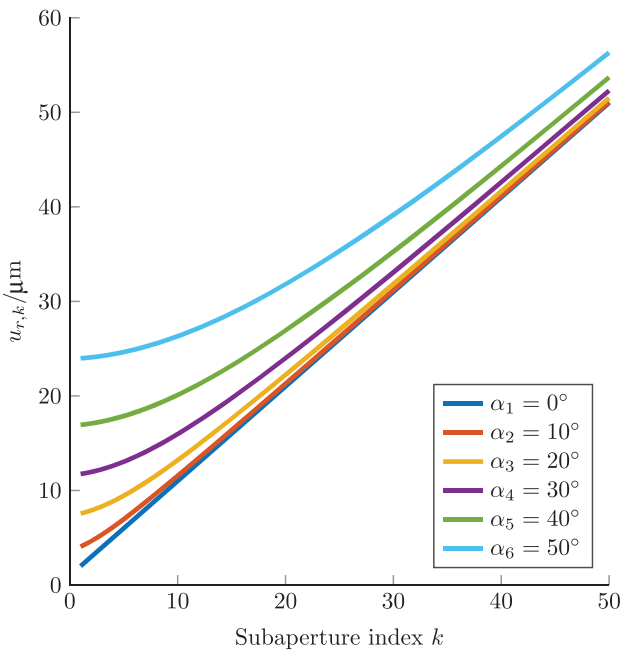

(a)

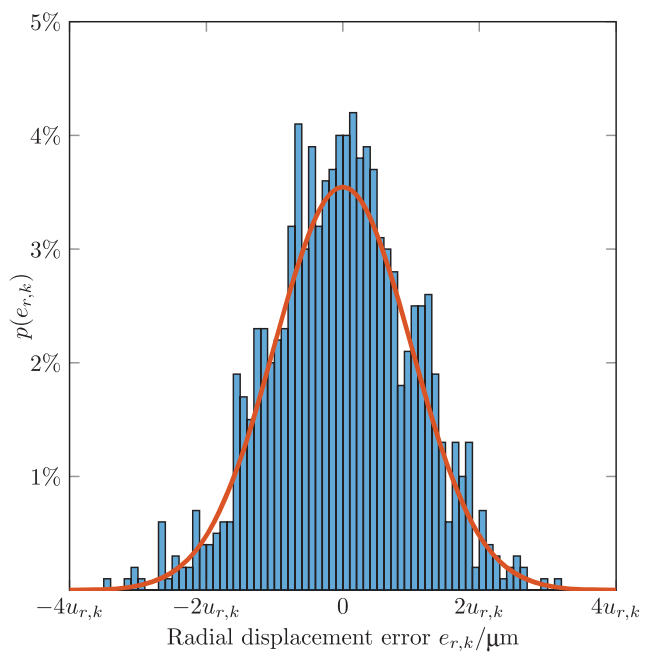

(b)

Fig. 3 (a) Radial uncertainty $u_{r, k}$ in dependence of the local tilt angle $\alpha_{k}$ and the number of subapertures $k \in[1, \cdots, K]$. (b) Probability density function $p\left(e_{r, k}\right)$ (orange solid line) of the simulated normally distributed lateral displacement error $e_{r, k}$ and the histogram of $N=1000$ computer generated samples based on $p\left(e_{r, k}\right)$.

$$
\begin{aligned}
& \Delta z_{k}=\left[z_{k}\left(r_{k 0}\right)-\overline{z_{k}}\left(r_{k 0}\right)\right]-\left[z_{k}\left(r_{k 1}\right)-\overline{z_{k}}\left(r_{k 1}\right)\right] \\
& e_{\alpha, k} \approx \operatorname{atan}\left(\frac{\Delta z_{k}}{\left|r_{k 1}-r_{k 0}\right|_{\perp}}\right) \\
& e_{r, k} \approx\left(L_{\text {ref }}+L_{\text {rot }}\right) \sin \left(e_{\alpha}\right) .
\end{aligned}
$$

The interferometric line sensor is employed to measure $K=8$ subapertures on the surface of a spherical reference object with radius of curvature $R=150 \mathrm{~mm}$. The measurement is repeated $N=20$ times, and the recorded topography sections $z_{k}$ are shown in Fig. 4(a) in comparison to the mean topography profile $\overline{z_{k}}$. Figure 4(b) shows the deviation of the measured topography $z_{k}$ to the mean topography $\overline{z_{k}}$, which is employed in Eq. (8) to get an estimate of the associated lateral displacement error. The radius of curvature of the considered sphere is $R=150 \mathrm{~mm}$ and the highest tilt angle in the field of view is $\alpha_{8} \approx 6.5 \mathrm{deg}$. The field of view is covered by $K=9$ subapertures with the outer radius being $r_{71} \approx 20 \mathrm{~mm}$. The modeled lateral displacement uncertainty $u_{r, k}$, according to Fig. 3(a), is depicted in Fig. 5 compared to the empirical standard deviation of the $N=20$ measurements for each subaperture $k \in[0, \cdots, 7]$. The comparison of the modeled uncertainty to the empirical standard deviation in Fig. 5 indicates that the parameters of the model have to be adapted for the first few subapertures, to expect a higher uncertainty. For higher subaperture counts, the worst case estimation of the $x$-axis position uncertainty $\left[(k+1) \cdot u_{x, \mathrm{it}}\right]$ is too high. However, the comparison shows that the modeled uncertainty is in the correct order of magnitude and may yield improved estimates of the measurement uncertainty adapting the parameters of Eq. (7).

The lateral displacement errors in the azimuthal direction depend on the uncertainty $u_{\theta}$ of the rotation stage $\mathrm{C}$, which is specified by the supplier as $u_{\theta} \approx 2^{\prime \prime}$. Considering a specimen diameter of $D \leq 300 \mathrm{~mm}$, this results in a maximum azimuthal displacement of $\pi D \cdot \frac{u_{\theta}}{360^{\circ}} \approx 1.5 \mu \mathrm{m}$. Since this deviation is smaller than the resolution of the employed $5 \times$ microscope objective of numerical aperture NA $=0.14$ and does not increase with the number of subapertures, it is neglected. The virtual experiments introduced in Sec. 4 will demonstrate the propagation of the lateral displacement errors $e_{r, k}$ in radial direction covered by the combined uncertainty $u_{r, k}$ to the topography results after stitching of the subapertures. The Monte-Carlo simulation employs the probability density function of normally distributed lateral displacement errors with mean $\mu=0$ and standard deviation $\sigma=u_{r, k}$ as depicted in Fig. 3(b). Randomization software is employed to generate samples $e_{r, k}$ based on the probability density function $p\left(e_{r, k}\right)$. A set of $N=1000$ samples is illustrated by the histogram in Fig. 3(b). The stitching algorithms employed in the virtual 


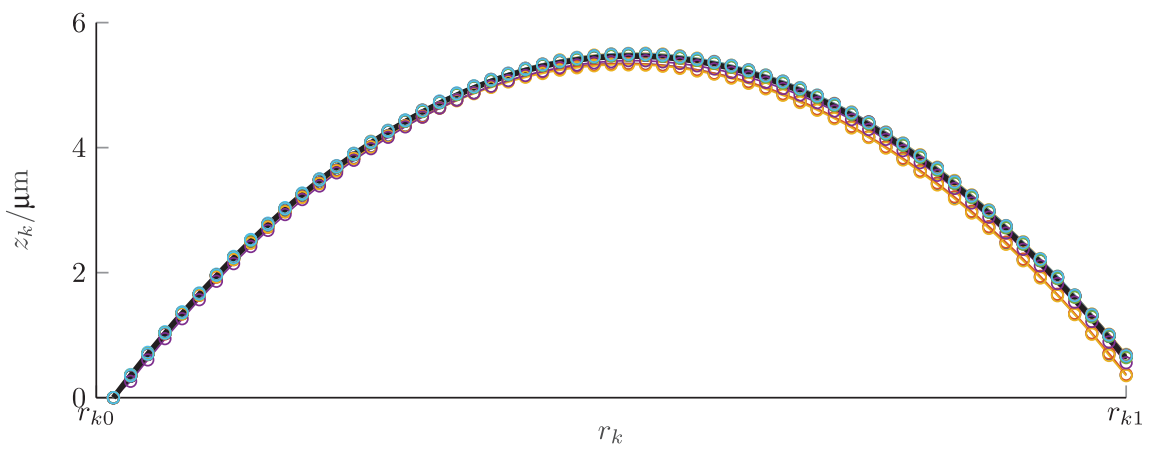

(a)

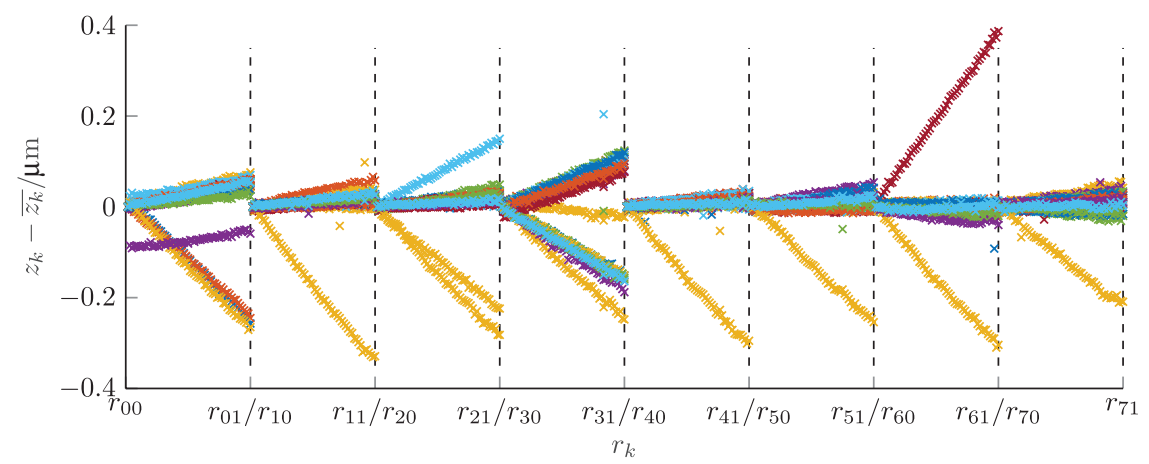

(b)

Fig. 4 (a) $N=20$ repetition measurements of a single subaperture section $z_{k}$ (colored rings) and the mean value of the repetition measurements $\overline{z_{k}}$ (black line). (b) Deviation of the measured topography $z_{k}$ to the mean topography $\overline{z_{k}}$, indicating statistical deviations. For the measurement, 1200 pixel are employed, yielding $\left|r_{k 1}-r_{k 0}\right|_{\perp} \approx 2.5 \mathrm{~mm}$. The subapertures overlap but are depicted separated on their radial intervals. The field of view is limited by $r_{71} \approx 20 \mathrm{~mm}$.

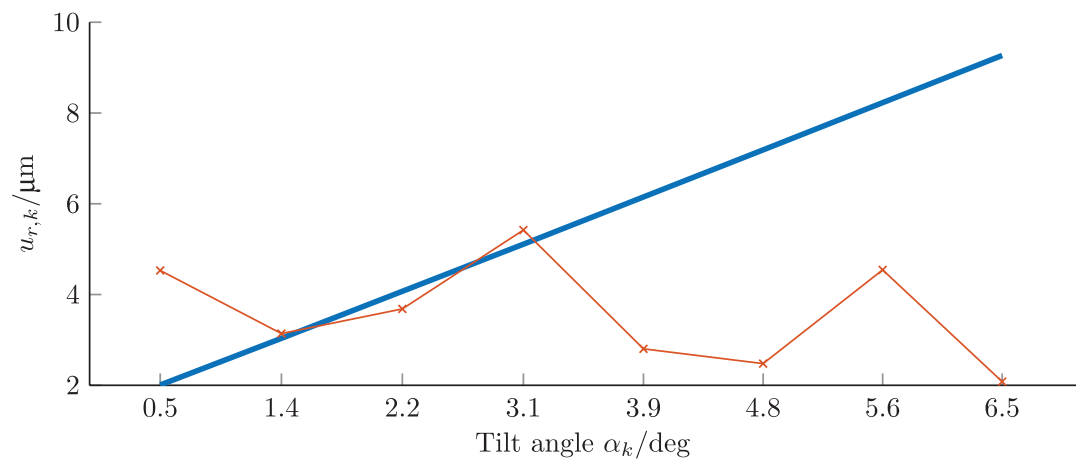

Fig. 5 Comparison of the modeled uncertainty $u_{r, k}$ (blue line) to the empirical standard deviation of the measurements (orange crosses).

experiment are designed to handle the topography measurements of an interferometer without tilting. Thus, they require the local topographies to show a correctly retrieved slope on the subapertures as depicted in Fig. 9(a). At the current state of the setup, this precludes the application of the stitching algorithms to evaluate interferograms recorded with the rotatable interferometric line sensor. The functionality of the stitching algorithms is extended to be capable to handle the interferograms recorded by the interferometric setup. However, the basic implementation of the virtual experiment is outlined in Sec. 4 to present the idea of propagating the topography deviations caused by lateral displacement uncertainties through the stitching algorithms and yield an estimate of the global topography deviation. 


\section{Virtual Experiment}

The result of a complete topography measurement employing the annular subaperture interferometry method introduced above is an estimate of the global wavefront $W(r, \theta)$, which approximates the measurand $\tilde{W}(r, \theta)$ describing the stitched three-dimensional surface topography of the specimen. To employ the setup for calibrations, the topography estimate $W(r, \theta)$ needs to be assigned a statement of expanded uncertainty ${ }^{12}$

$$
U_{W}=k_{p} u_{W}
$$

The expanded uncertainty $U_{W}$ defines a confidence interval

$$
W(r, \theta)-U_{W} \leq \tilde{W}(r, \theta) \leq W(r, \theta)+U_{W}
$$

with a specific coverage probability $p$.

The above combination of a confidence interval with a certain level of confidence and an associated coverage probability is referred to as coverage interval and has the following meaning. Considering an experiment is repeated $N$ times yielding $N$ estimates of the measurand referred to as samples. Based on these $N$ samples, the probability density function of the sample distribution is retrieved and a confidence interval [as in Eq. (10)] is defined, which will contain a sample with a certain probability. The confidence level, indicated by the coverage factor $k_{p}$, yields the probability for each sample to lie within the confidence interval. If the set of $N$ experiments, which yields one probability density function and a confidence interval is repeated $Z$ times, it yields $Z$ probability density functions with $Z$ confidence intervals. The coverage probability states the probability for each of these confidence intervals to contain the actual measurand and thus, the probability of the experimental procedure to yield a reasonable result. The coverage probability is independent of the sample number $N$, but depends on the confidence level and the statistical distribution. In case of a normal distribution, the coverage probability converges to the confidence level. $^{12,19}$ A high confidence level (e.g., $\pm 3 \sigma, k_{p}=3, p=99.73 \%$ ) is associated with a broad confidence interval, which means a low accuracy but a high coverage probability. A low confidence level (e.g., $\pm \sigma, k_{p}=1, p=68.27 \%$ ) yields higher accuracy but a lower coverage probability.

The expanded uncertainty $U_{W}$ depends on the combined uncertainty $u_{W}$ and the coverage factor $k_{p}$ as given in Eq. (9). To obtain the coverage factor $k_{p}$, the probability distribution of the measurement results $W(r, \theta)$ with the combined uncertainty $u_{W}$ is required. The probability distribution of the measurement results $W(r, \theta)$ depends on the radial displacement errors, which are assumed to be normally distributed as depicted in Fig. 3(b), and which are characterized by the combined uncertainty $u_{r, k}$. However, the topography $W(r, \theta)$ has no linear dependence on the radial position $r$ since it propagates through the stitching algorithm. Thus, there is no convenient analytic approach available to obtain the probability distribution of the topography estimates $W(r, \theta)$ from the distribution of the radial displacement errors. Instead, a virtual experiment is employed that will simulate a topography measurement with $K$ subapertures. Based on the combined uncertainty $u_{r, k}$, a normally distributed radial displacement error is added to the radial intervals of each subaperture.

The expanded uncertainty $U_{W}$ and the combined uncertainty $u_{W}$ of the retrieved surface topography are estimated employing the distribution of the Zernike coefficients describing the surface form. The deviation in the Zernike coefficients is directly associated to a certain feature, e.g., piston, tilt, power, astigmatism, and so on, of the surface. Therefore, it yields information about the form of the topography deviation. Each topography result yields $L=36$ Zernike coefficients describing the estimate $W(r, \theta)=\mathbf{X} \cdot \mathbf{P}$ as introduced in Eq. (2). Other than for the global optimization problem in Eq. (2), there are no subapertures considered and the matrices $[\mathbf{X}]_{N_{\text {glo }} \times L}$ and $[\mathbf{P}]_{L \times 1}$ only represent the $N_{\text {glo }}$ sample points in the global coordinate system. The measurand $\tilde{W}(r, \theta)=\mathbf{X} \cdot \mathbf{P}_{\text {inp }}$, which is the input topography of the simulation before sampling of the subapertures and adding of the radial uncertainty, is also described by $L=36$ Zernike coefficients. In case of the global stitching, the first $M=3$ coefficients of piston and tilts equal 0 since they are reserved to describe the translational errors between the subapertures. In case of 
the cumulative stitching, the first $M=3$ coefficients describe the orientation of the subaperture employed as the basis for the subaperture stitching. This orientation may be arbitrary with respect to the global coordinate system and thus, does not contribute to the form information. The actual surface form is given by the $L-M$ coefficients of higher order. One virtual experimental set contains $N=10000$ simulations with normally distributed radial displacement errors as depicted in Fig. 3(b), yielding $N$ matrices $[\mathbf{P}]_{L \times 1}$ of Zernike coefficients. As outlined above, the combined uncertainty $u_{W}$ is estimated employing the distribution of form deviations represented by the Zernike coefficients $\mathbf{P}_{\text {glo }}$ of global and $\mathbf{P}_{\text {cum }}$ of cumulative stitching relative to the constant set of topography input parameters $\mathbf{P}_{\text {inp }}$.

The topography $W(r, \theta)$ and the expanded uncertainty $U_{W}$ also depend on the surface measurement results of the employed Michelson interferometer, which are influenced by electrical and thermal noise, wavelength instabilities, and mechanical vibrations. The uncertainty contributions of these effects are not covered by the virtual experiments in this contribution.

The virtual experiment employs a spherical specimen of radius $R_{\mathrm{sph}}=300 \mathrm{~mm}$, it emulates a measurement on the spherical copper specimen depicted in Fig. 1(a). The region of interest, which is centered on the spherical specimen, has a diameter of $D=200 \mathrm{~mm}$ and is sampled in $K=14$ overlapping subapertures. A set of $L=36$ Zernike polynomial coefficients is employed to describe the input surface profile and to fit the stitched output topography. The translational errors due to wavefront aberrations between the subapertures are described employing the first $M=3$ Zernike coefficients of piston and two tilts, and the subapertures employed in the cumulative stitching approach are modeled employing $L_{\text {sub }}=21$ Zernike coefficients. The number of employed Zernike coefficients for the topography fitting affects the results. If the number of coefficients is chosen insufficiently high for the simulated smooth surface sections of the spherical object, badly scaled matrices appear in the optimization problem and cause numerical errors. The nominal length of the line sensor array imaged on the specimen by the $5 \times$ microscope objective is $\left|r_{k 1}-r_{k 0}\right|_{\perp} \approx 8.65 \mathrm{~mm}$, if oriented perpendicular to the specimen surface. The radial intervals $r_{k}=\left[r_{k 0}, r_{k 1}\right]$ covered by the $k^{\prime}$ th subaperture, in dependence of the local tilt angle, are calculated employing the normal distributed uncertainty $u_{r, k}$ as outlined above. The simulated input topography, which is described by the Zernike coefficients $\mathbf{P}_{\text {inp }}$ is depicted in Fig. 6(a). Figure 6(b) shows an example of the form deviation between the input topography and the stitched topography for a single iteration of the virtual experiment. To identify the probability distribution function of the combined uncertainty $u_{W}, N=10000$ topography samples are generated employing the normal distributed radial displacement uncertainty $u_{r, k}$ and the deviation in

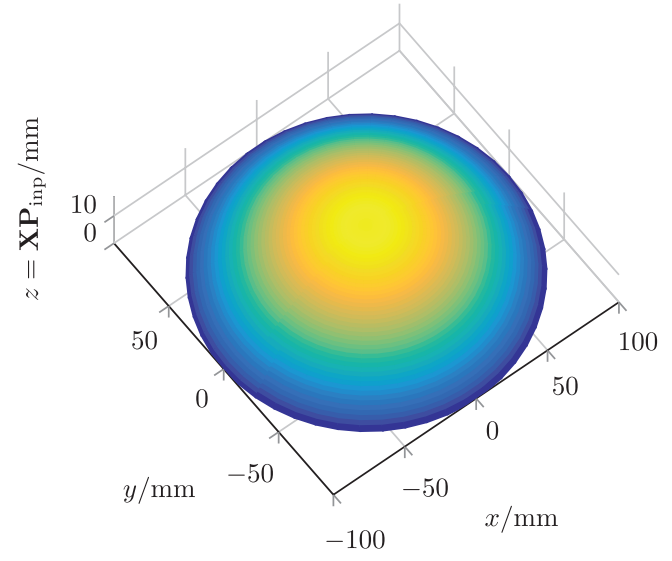

(a)

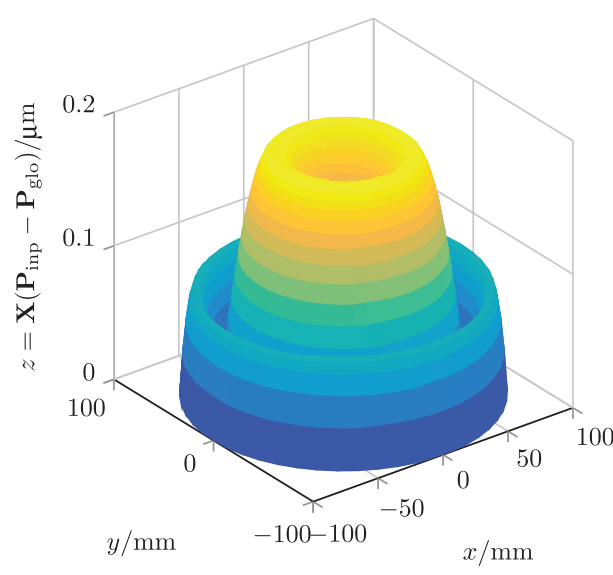

(b)

Fig. 6 (a) Simulated section $D=200 \mathrm{~mm}$ of the topography of a spherical, polished copper specimen of radius $R_{\mathrm{sph}}=300 \mathrm{~mm}$, described by the Zernike coefficient vector $\mathbf{P}_{\text {inp }}$ evaluated for a set of $N_{\text {glo }}$ topography points. (b) Example of the topography difference between the input topography and the topography retrieved by global stitching employing the radial displacement uncertainty $u_{r, k}$ and $K=14$ subapertures for one of the $N=10000$ virtual experiments. 
Table 2 Zernike annular polynomials $Z_{k i}\left(r_{k}, \theta, k\right)$, with $i$ counting the coefficients and $k$ indicating the subaperture index. The indices $n_{z}$ and $m_{z}$ are employed in the iterative calculation of the Zernike polynomials. ${ }^{14}$

\begin{tabular}{cccc}
\hline \hline$i$ & $n_{z}$ & $m_{z}$ & Meaning \\
\hline 1 & 0 & 0 & Piston \\
2 & 1 & -1 & $y$-tilt \\
3 & 1 & 1 & $x$-tilt \\
4 & 2 & -2 & Astigmatism \\
5 & 2 & 0 & Power/defocus \\
6 & 2 & 2 & Astigmatism \\
\hline \hline
\end{tabular}

the Zernike coefficient vectors $\mathbf{P}_{\text {inp }}-\mathbf{P}_{\text {glo }}$ and $\mathbf{P}_{\text {inp }}-\mathbf{P}_{\text {cum }}$ are analyzed for each coefficient. Since the input topography is a sphere cap, one of the main contributions to the topography form is described by the Zernike coefficient $\mathbf{P}(5)$ associated with the radius, referred to as defocus or power. Table 2 gives an oversight how the Zernike polynomials are counted in this contribution and what topography features are associated with the coefficients. The empirical probability density function of the deviation $e_{\mathbf{P}_{\text {glo }} \text { (5) }}=\mathbf{P}_{\text {inp }}(5)-\mathbf{P}_{\text {glo }}(5)$ and a normal distribution based on the uncertainty $u_{\mathbf{P}_{\text {glo }}(5)}$ is shown in Fig. $7(\mathrm{a})$. The empirical standard deviation (standard uncertainty) $u_{\mathbf{P}_{\text {glo }} \text { (5) }}$ is calculated based on the empirical distribution of $e_{\mathbf{P}_{\text {glo }} \text { (5) }}$ as follows:

$$
\begin{gathered}
\mu_{e_{\mathbf{P}_{\mathrm{glo}}(5)}}=\frac{1}{N} \sum_{n=1}^{N} e_{\mathbf{P}_{\mathrm{glo}}(5)}(n), \\
u_{\mathbf{P}_{\mathrm{glo}}(5)}=\sqrt{\frac{1}{N-1} \sum_{n=1}^{N}\left(e_{\mathbf{P}_{\mathrm{glo}}(5)}(n)-\mu_{e_{\mathbf{P}_{\mathrm{glo}}(5)}}\right)^{2}} .
\end{gathered}
$$

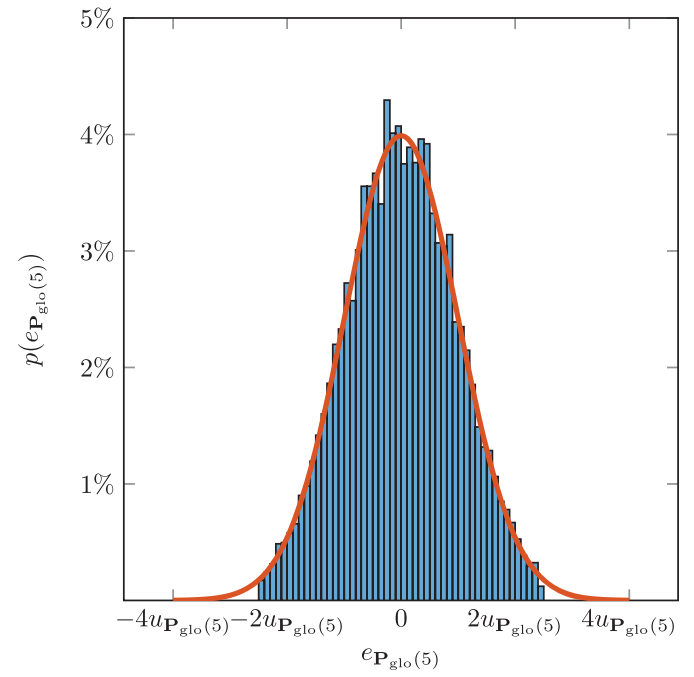

(a)

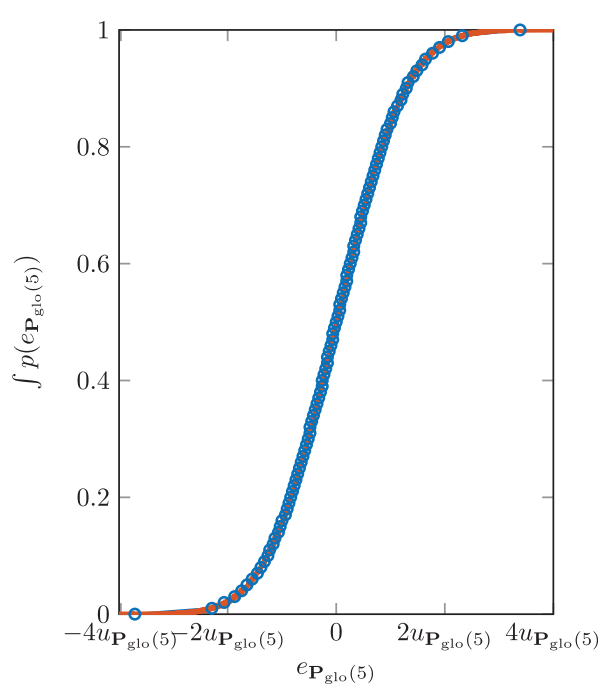

(b)

Fig. 7 (a) Histogram representation of the empirical deviation in the Zernike polynomials power term $e_{\mathbf{P}_{\mathrm{glo}}(5)}$ for $N=10000$ samples, normal distribution based on the standard uncertainty $u_{\mathbf{P}_{\mathrm{glo}}(5)}$ (solid orange line). (b) Cumulative empirical distribution function (blue circles) and cumulative probability distribution of the normal distribution $\mathbf{P}_{\mathrm{glo}}(5)$ (solid orange line). 

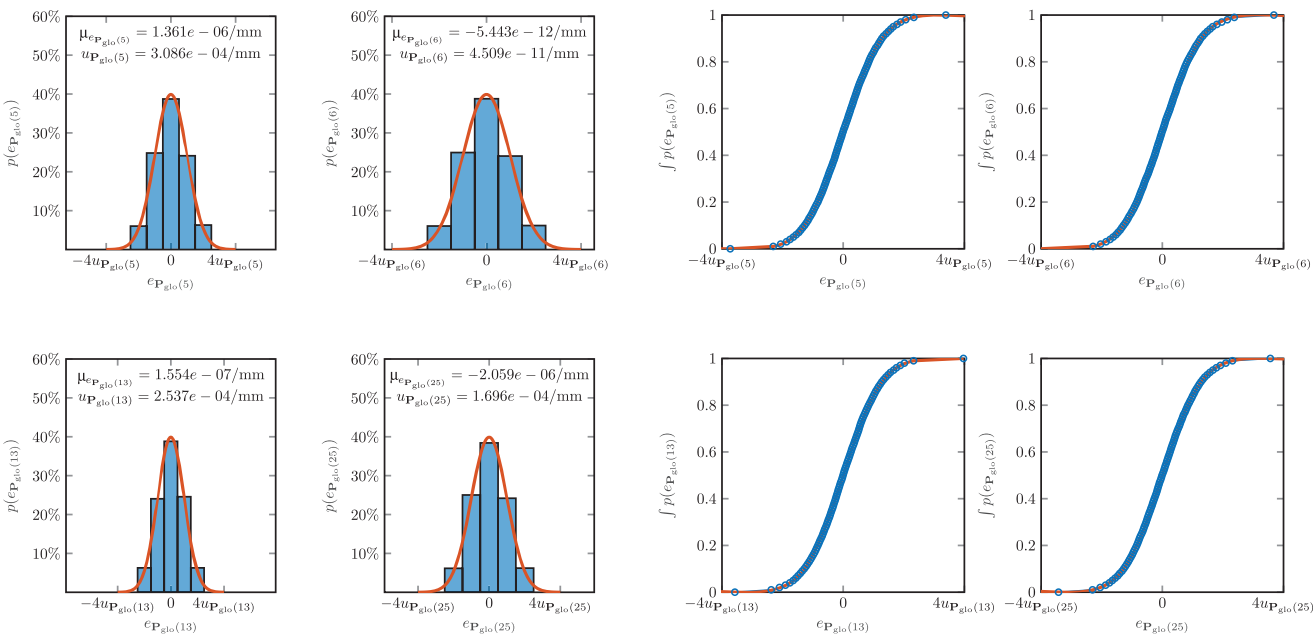

(a)

(b)

Fig. 8 (a) Histogram representation of the empirical deviation in the dominant Zernike polynomial terms $e_{\mathrm{P}_{\mathrm{glo}}(i)}, i \in[5,13,25]$ and a neglectable small coefficient $i=6$ employing $N=10000$ samples, normal distribution based on the standard uncertainty $u_{\mathrm{P}_{\mathrm{glo}}(i)}$ (solid orange line). (b) Cumulative empirical distribution function (blue circles) and cumulative probability distribution of the normal distribution $p\left(e_{\mathbf{P}_{\mathrm{glo}}(i)}\right)$ (solid orange line).

The cumulative empirical distribution function (blue circles) and cumulative probability distribution of the normal distribution $\mathbf{P}_{\text {glo }}(5)$ (solid orange line) in Fig. 7(b) indicate that the deviations in the Zernike polynomial coefficient $\mathbf{P}_{\text {glo }}(5)$ are normally distributed. Figure 8 shows the empirical distribution functions of the $i$ 'th Zernike coefficients deviation $e_{\mathbf{P}_{\text {glo }}(i)}=$ $\mathbf{P}_{\text {inp }}(i)-\mathbf{P}_{\text {glo }}(i)$ with $i \in[5,6,13,25]$ in comparison to a normal distribution. The distribution functions of the deviations $e_{\mathbf{P}_{\text {glo }}(i)}$ and $e_{\mathbf{P}_{\text {cum }}(i)}$ are normal distributed for all $i \in[M+1, \cdots, L]$ Zernike coefficients describing the surface form. Therefore, it may be concluded that the expanded uncertainty $U_{W}$ in dependence of the radial displacement errors is also normal distributed and may be estimated employing the following equation:

$$
U_{W} \approx k_{p} \mathbf{X} \cdot \vec{u}_{\mathbf{P}_{\mathrm{glo}}} .
$$

With $\vec{u}_{\mathbf{P}_{\text {glo }}}$ being an $L \times 1$ vector containing the combined standard uncertainty $u_{\mathbf{P}_{\text {glo }}}(i)$ of each Zernike coefficient. This representation of the expanded uncertainty $U_{W}$ assigns a confidence interval with a coverage probability given by $k_{p}$ to each point on the topography depending on its lateral coordinates similar to the deviation map in Fig. 6(b). This yields detailed information about the local uncertainty on the specimen while considering the same field of view on the specimen represented by the matrix $\mathbf{X}$ reducing the comparison of the topography to a comparison of the Zernike coefficients. Yet, the expanded uncertainty $k_{p} \cdot \vec{u}_{\mathbf{P}_{\text {glo }}}$ alone lacks a representation in terms of the SI unit of length, which is added in Eq. (13) by multiplication with $\mathbf{X}$ containing the value of the Zernike polynomial evaluated at the supporting point $(r, \theta)$. However, if the Zernike coefficients are scaled to the diameter of the field of view $D$ and $\mathbf{X}$ is defined on the unit circle, which yields a straight forward calculation independent of the diameter, the coefficients may be assigned a unit of length as done in Fig. 8(a) and Table 3. In addition to $\vec{u}_{\mathbf{P}_{\text {glo }}}$, the uncertainty in the Zernike coefficients, it is convenient to employ the peak-to-valley (PV) deviation of the expanded uncertainty $U_{W}$, which represents the expected maximum absolute deviation between the measurand $\tilde{W}(r, \theta)$ and its estimate $W(r, \theta)$.

The results of the virtual experiment in Table 3 indicate that the 5th, 13th, and 25th Zernike coefficient are the dominant source of deviations for the considered spherical specimen, when employing $L=36$ Zernike coefficients. This is reasonable since the associated Zernike polynomials $Z_{0}^{2}, Z_{0}^{4}$, and $Z_{0}^{6}$ are rotationally invariant as is the considered specimen. The neglectable small uncertainties of the other coefficients are most probably caused by numeric errors during 
Table 3 Results of the virtual experiment for the expanded uncertainty estimates employing different $k_{p}$ values and $L=36$ Zernike coefficients.

\begin{tabular}{|c|c|c|c|c|}
\hline$i$ & 5 & 6 & 13 & 25 \\
\hline \multicolumn{5}{|l|}{$L=36$} \\
\hline $\mathbf{P}_{\mathrm{inp}}(i) / \mathrm{mm}$ & $-8.58 e+00$ & $-8.41 e-13$ & $-8.41 e-02$ & $-1.47 e-03$ \\
\hline$\vec{\mu}_{\mathbf{P}_{\mathrm{glo}}}\left(i, k_{p} u_{r, k}\right) / \mathrm{mm}, k_{p}=1$ & $-8.58 e+00$ & $-7.39 e-12$ & $-8.41 e-02$ & $-1.49 e-03$ \\
\hline$\vec{u}_{\mathrm{P}_{\mathrm{glo}}}\left(i, k_{p} u_{r, k}\right) / \mathrm{mm}, k_{p}=1$ & $3.09 e-04$ & $4.51 e-11$ & $2.54 e-04$ & $1.70 e-04$ \\
\hline$\vec{u}_{\mathrm{P}_{\mathrm{glo}}}\left(i, k_{p} u_{r, k}\right) / \mathrm{mm}, k_{p}=2$ & $6.15 e-04$ & $8.80 e-11$ & $4.96 e-04$ & $3.32 e-04$ \\
\hline $\mathrm{PV}\left\{U_{W_{\mathrm{glo}}(i)}\right\} / \mathrm{mm}, k_{p}=1$ & $6.17 e-04$ & $9.02 e-11$ & $3.81 e-04$ & $3.39 e-04$ \\
\hline $\mathrm{PV}\left\{U_{W_{\mathrm{glo}}(i)}\right\} / \mathrm{mm}, k_{p}=2$ & $1.23 e-03$ & $1.76 e-10$ & $7.44 e-04$ & $6.65 e-04$ \\
\hline$\vec{\mu}_{\mathbf{P}_{\text {cum }}}\left(i, k_{p} u_{r, k}\right) / \mathrm{mm}, k_{p}=1$ & $-8.58 e+00$ & $-3.19 e-11$ & $-8.41 e-02$ & $-1.49 e-03$ \\
\hline$\vec{u}_{\mathrm{P}_{\text {cum }}}\left(i, k_{p} u_{r, k}\right) / \mathrm{mm}, k_{p}=1$ & $3.08 e-04$ & $4.70 e-10$ & $2.38 e-04$ & $2.75 e-04$ \\
\hline$\vec{u}_{\mathrm{P}_{\text {cum }}}\left(i, k_{p} u_{r, k}\right) / \mathrm{mm}, k_{p}=2$ & $6.14 e-04$ & $9.29 e-10$ & $4.64 e-04$ & $5.36 e-04$ \\
\hline $\operatorname{PV}\left\{U_{W_{\text {cum }}(i)}\right\} / \mathrm{mm}, k_{p}=1$ & $6.16 e-04$ & $9.40 e-10$ & $3.57 e-04$ & $5.50 e-04$ \\
\hline $\operatorname{PV}\left\{U_{W_{\text {cum }}(i)}\right\} / \mathrm{mm}, k_{p}=2$ & $1.23 e-03$ & $1.86 e-09$ & $6.96 e-04$ & $1.07 e-03$ \\
\hline
\end{tabular}

the optimization procedure when the Zernike coefficients are retrieved by solving the linear optimization problem. ${ }^{9,10,18}$ The results also indicate that with respect to the propagation of radial displacement errors through the stitching procedure there is no remarkable difference between the global- and the cumulative-stitching approach. To obtain a worst case estimation of the form deviation, the sum of the PV values over all $L$ Zernike coefficients is considered.

$$
\operatorname{PV}\left\{U_{W}\right\}=\sum_{i=1}^{L} \operatorname{PV}\left\{U_{W}(i)\right\}
$$

Since the numeric values presented in Table 3 are calculated based on the stitching algorithms for the interferometric signals depicted in Fig. 9(a) and the actual experiment employs the signals

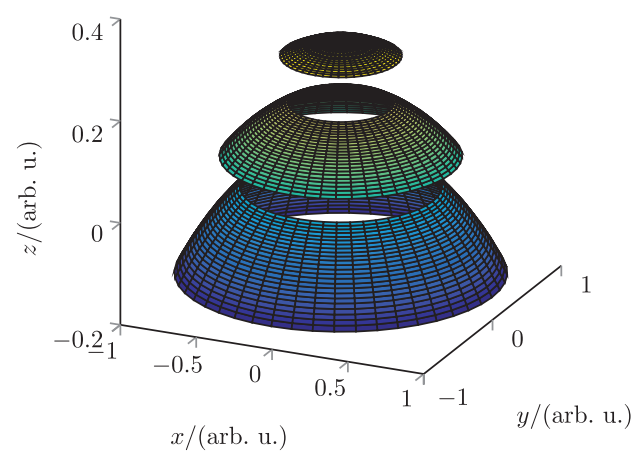

(a)

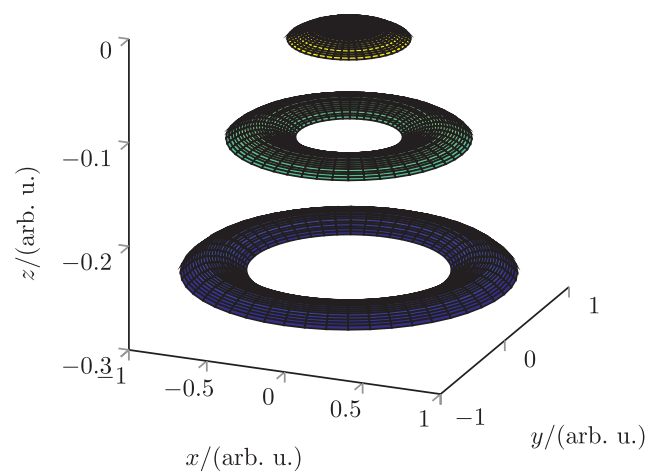

(b)

Fig. 9 (a) Sampled subaperture rings with correctly retrieved slope in global coordinates. (b) Sampled subaperture rings in local coordinates, where the slope information is lost due to the tilting of the interferometer. The slope information may be restored from the overlapping regions or by realignment of the rings in a global optimization problem in Cartesian coordinates. 
depicted in Fig. 9(b), they are not significant to predict the achievable uncertainty of the measurement setup. However, the interesting result of the virtual experiment is the observation that a normally distributed lateral displacement error in the local topographies causes a normally distributed deviation in the global topography. The deviation may be modeled by the uncertainty of the Zernike coefficients used to describe the global topography and yields a compact positiondependent description of the measurement uncertainty. The extended stitching algorithms will be based on the same principle as those employed in the presented virtual experiment and thus are expected to show a similar behavior.

\subsection{Limitations of the Model}

The simulation results obtained by the virtual experiment and the stitching algorithms presented in Refs. 9 and 10 are based on the assumption that the subaperture rings of the spherical or aspherical specimen under consideration are obtained with a correct slope, such as if the topography in global coordinates is sliced into multiple rings, as illustrated in Fig. 9(a). These rings may have an arbitrary offset or tilt to each other and may be realigned by compensation of these $M=3$ low-order translational errors. However, preliminary measurement results obtained by the interferometer show that the single rings are measured as pole caps in the local coordinate system due to the tilting of the interferometric sensor with respect to the specimen, as illustrated in Fig. 9(b). Therefore, the translational errors in the overlapping areas of the subapertures may not be described by the Zernike polynomials of piston and tilt, but take the form of tilted rings in polar coordinates. Transforming these rings into Cartesian coordinates reduces the complexity of the translational errors to offset and tilt, and a similar stitching procedure may be applied employing Cartesian polynomials. The main difference to the virtual experiment is that in the practical realization the reference wavefronts of each local subaperture are tilted to each other, due to the tilting of the interferometer and therefore, the slope of each ring in global coordinates has to be restored, either employing the absolute distance of the center of rotation to the interferometer $L_{\text {ref }}+L_{\text {rot }}$ and the angle $\alpha_{k}$ of the tilting axes or by realignment of the subapertures employing the information of the overlapping regions or solving a global optimization problem in Cartesian coordinates. Employing the enhanced stitching procedure in the virtual experiment, it should be possible to estimate the statistical topography deviations based on the statistical lateral displacement errors. However, the model does currently not include electrical and thermal noise, wavelength instabilities, and mechanical vibrations, which also contribute to the statistical error. And it does not model possible systematic errors, which occur when the probe is moved to the next subaperture employing an erroneous tilt angle for the calculation of the radial interval or topography measurement deviations caused by aberrations in the imaging optics.

\section{Conclusion}

This contribution considers the modeling of statistical lateral displacement errors $e_{r, k}$ in annular subaperture stitching interferometry (ASSI). Section 3 introduces a method to model the combined standard uncertainty $u_{r, k}$ of lateral displacement errors given by Eq. (7). A series of calibration measurements indicate that the statistical lateral displacement errors occurring during repositioning of the interferometric sensor between subaperture measurements are feasibly modeled as a normal distribution. The normal distribution of the lateral displacement errors in radial direction is characterized by the combined standard uncertainty $u_{r, k}$. A virtual experiment is employed to yield an estimate of the expanded standard uncertainty $U_{W}$, in dependence of the normally distributed lateral displacement errors $e_{r, k}$. The results of this virtual experiment indicate that the standard uncertainty $\vec{u}_{\mathbf{P}_{\text {glo }}}$ in combination with the coverage factor $k_{p}$ and the considered region of interest yields a suitable estimate of the expanded standard uncertainty as given in Eq. (13). The deviations in the Zernike coefficients describing the reconstructed topography in comparison to the simulated input topography $e_{\mathbf{P}_{\text {glo }}(i)}=\mathbf{P}_{\text {inp }}(i)-\mathbf{P}_{\text {glo }}(i)$ are normally distributed based on the set of $N=10000$ topography samples, generated in the virtual experiment. They are characterized by the standard uncertainty $u_{\mathbf{P}_{\text {glo }}}(i)$. Thus, the expanded standard uncertainty $U_{W}$ is a normally distributed topography deviation and the coverage factor $k_{p}$ may be 
calculated based on the desired coverage probability $p$ (e.g., $p=95.45 \%$ for $k_{p}=2$ ). Furthermore, the results presented in Table 3 indicate that a scaling of the lateral displacement uncertainty $k_{p} u_{r, k}$ transfers linear to the topography uncertainty $\vec{u}_{\mathbf{P}_{\text {glo }}}$. The introduced method for the estimation of an expanded standard uncertainty by propagating the lateral displacement errors of the ASSI setup through the virtual experiment yields a convenient tool to assert a statement of expanded uncertainty to the topography results, which will be retrieved by measurements with the annular subaperture stitching interferometer. Furthermore, the use of Zernike polynomial coefficients allows the comparison of variable regions of interest and the storing of the topography information in a highly compressed format while also allowing to evaluate the uncertainty contribution of single coefficients. However, the comparison of the modeled lateral displacement error to the statistical deviations of subaperture measurements presented in Fig. 5 indicates that the parameters of the radial displacement uncertainty require further adaptations. Yet, the distribution of the radial displacement errors could be characterized. In future work, the results of the virtual experiment will be compared to actual topography measurement results and the influence of additional sources of uncertainty, mostly related to the interferometric measurements with the employed Michelson interferometer, which are influenced by electrical and thermal noise, wavelength instabilities, and mechanical vibrations are considered.

\section{Acknowledgments}

The authors gratefully acknowledge the financial support for this research project (Award Nos. LE 992/7-2 and EH 400/4-2) received from the Deutsche Forschungsgemeinschaft (DFG).

\section{References}

1. M. Eifler et al., "Calibration sample for arbitrary metrological characteristics of optical topography measuring instruments," Opt. Express 26, 16609-16623 (2018).

2. R. Leach, "Some issues of traceability in the field of surface topography measurement," Wear 257(12), 1246-1249 (2004).

3. I. Fortmeier, M. Schulz, and R. Meeß, "Traceability of form measurements of freeform surfaces: metrological reference surfaces," Opt. Eng. 58(9), 092602 (2019).

4. A. J. Henning and C. L. Giusca, "Errors and uncertainty in the topography gained via frequency-domain analysis," Opt. Express 23, 24057-24070 (2015).

5. I. Fortmeier et al., "Evaluation of absolute form measurements using a tilted-wave interferometer," Opt. Express 24, 3393-3404 (2016).

6. H. Knell et al., "Continuous measurement of optical surfaces using a line-scan interferometer with sinusoidal path length modulation," Opt. Express 22, 29787-29798 (2014).

7. S. Laubach et al., "Combination of a fast white-light interferometer with a phase shifting interferometric line sensor for form measurements of precision components," Proc. SPIE 10329, 103291D (2017).

8. J. Riebeling, G. Ehret, and P. Lehmann, "Optical form measurement system using a linescan interferometer and distance measuring interferometers for run-out compensation of the rotational object stage," Proc. SPIE 11056, 110562D (2019).

9. M. Schake and G. Ehret, "Annular subaperture stitching interferometry with planar reference wavefront for measurement of spherical and aspherical surfaces," Proc. SPIE 11523, 115230D (2020).

10. M. Schake and G. Ehret, "Machine learning based fitting of Zernike polynomials for ASSI," in DGaO-Proc. 2020, Vol. 121(A31), Paper DGaO (2020).

11. M. Schulz and P. Lehmann, "Measurement of distance changes using a fibre-coupled common-path interferometer with mechanical path length modulation," Meas. Sci. Technol. 24(6), 065202 (2013).

12. Joint Committee for Guides in Metrology, "Evaluation of measurement data-guide to the expression of uncertainty in measurement," JCGM 100:2008, BIPM Joint Committee for Guides in Metrology, Paris, 2008, https://www.bipm.org/utils/common/documents/jcgm/ JCGM_100_2008_E.pdf (accessed 27 April 2020). 
13. A. K. Bewoor and V. A. Kulkarni, Metrology \& Measurement, 1st ed., McGraw Hill, India (2009).

14. X. Hou et al., "Full-aperture wavefront reconstruction from annular subaperture interferometric data by use of Zernike annular polynomials and a matrix method for testing large aspheric surfaces," Appl. Opt. 45, 3442-3455 (2006).

15. M. F. Küchel, "Interferometric measurement of rotationally symmetric aspheric surfaces," Proc. SPIE 7389, 738916 (2009).

16. X. Wang et al., "Measurement of large aspheric surfaces by annular subaperture stitching interferometry," Chin. Opt. Lett. 5(11), 645-647 (2007).

17. Y. Wen et al., "Modified stitching algorithm for annular subaperture stitching interferometry for aspheric surfaces," Appl. Opt. 52, 5686-5694 (2013).

18. D. R. Ibañez et al., "Robust fitting of Zernike polynomials to noisy point clouds defined over connected domains of arbitrary shape," Opt. Express 24, 5918-5933 (2016).

19. University of Oxford Department of Statistics, "Descriptive statistics for research," Hilary Term, 2002, IAUL Department of Statistic, Oxford, 2002, https://www.stats.ox.ac.uk/pub/ bdr/IAUL/Course1Notes5.pdf (accessed 26 May 2020).

Markus Schake received his $\mathrm{PhD}$ in electrical engineering from the University of Kassel in 2019. He currently works as a researcher at Physikalisch-Technische Bundesanstalt (PTB). His research interests include interferometry, topography measurement, frequency-domain analysis, non-uniform wave propagation, and topography stitching.

Jörg Riebeling is working as a PhD student at the measurement technology group of the University of Kassel. His research interests are focused in optical metrology, development of software components for real-time evaluation, topography measurement, and frequency-domain analysis.

Gerd Ehret is the head of the PTB working group "Flatness Metrology" and has set up a globally unique flatness measurement system with nanometre uncertainty. He investigates promising new interferometric and deflectometric approaches to measure the form also from curved surfaces with high accuracy. He has more than 15 years' experience in optical metrology (optical flatness/form metrology, wavefront metrology, optical microscopy, optical rigorous modelling, improving of optical antireflexion, shearing technology) and published more than 50 scientific papers in this field. 